\title{
Escala de Percepção do Suporte Social (versão adulta) - EPSUS-A: estudo das qualidades psicométricas
}

\author{
Hugo Ferrari Cardoso - Universidade São Francisco, Itatiba, Brasil \\ Makilim Nunes Baptista - Universidade São Francisco, Itatiba, Brasil
}

\begin{abstract}
Resumo
A presente pesquisa teve como objetivo realizar estudos de evidência de validade com base na estrutura interna e confiabilidade por meio do índice alfa de Cronbach $(\alpha$ ) para a Escala de Percepção do Suporte Social (versão adulta) - EPSUS-A. A amostra do estudo foi composta por 533 universitários, provenientes de três estados brasileiros. Procedeu-se à análise fatorial da EPSUS-A, inicialmente composta por 77 itens, e, por meio dessa, foi possível evidenciar validade com base na estrutura interna para a EPSUS-A. Nesse sentido, a escala ficou composta por 36 itens, aglutinados em quatro fatores, quais sejam, afetivo (composto por 17 itens e $\alpha=0,92$ ); interações sociais (cinco itens e $\alpha=0,75$ ); instrumental (sete itens e $\alpha=0,82$ ) e enfrentamento de problemas (sete itens e $\alpha=0,83$ ). A EPSUS-A se mostrou como uma escala adequada para avaliação do suporte social; entretanto, novos estudos são necessários no intuito de evidenciar outras formas de validade para o instrumento.

Palavras-chave: Avaliação psicológica; Testes psicológicos; Interação interpessoal.
\end{abstract}

\section{Scale of Perceived Social Support (adult version) - EPSUS-A: study of psychometric qualities}

\begin{abstract}
This research aimed to conduct studies of validity evidence based on internal structure and reliability through Cronbach alpha index $(\alpha)$ for the Scale of Perceived Social Support (old version) - EPSUS-A. The study sample consisted of 533 students from three states. Proceeded to the factor analysis of EPSUS-A initially composed of 77 items and, through this, it was possible to demonstrate validity based on internal structure for EPSUS-A. In this sense, the scale was composed of 36 items, clumped into four factors, namely, affective (composed of 17 items and $\alpha=0.92$ ); social interactions (five items and $\alpha=0.75$ ); instrumental (seven items and $\alpha=0.82$ ) and coping with problems (seven items and $\alpha=0.83$ ). The EPSUS-A was shown to be an appropriate scale for the assessment of social support, however further studies are needed in order to highlight other forms of validity for the instrument.

Keywords: Psychological assessment; Psychological tests; Interpersonal interaction.
\end{abstract}

\section{Escala de Apoyo Social Percibido (versión para adultos) - EPSUS-A: el estudio de las propiedades psicométricas}

\begin{abstract}
Resumen
Esta investigación tuvo como objetivo realizar estudios de evidencia de validez sobre la base de la estructura interna y la fiabilidad a través de índice alfa de Cronbach $(\alpha)$ de la Escala de Apoyo Social Percibido (versión antigua) - EPSUS-A. La muestra del estudio consistió en 533 estudiantes de tres estados. Procedió a análisis factorial de EPSUS-A inicialmente compuesto por 77 artículos y, a través de esto, fue posible demostrar la validez basada en la estructura interna de EPSUS-A. En este sentido, la escala se compone de 36 artículos, agrupadas en cuatro factores, a saber, afectivos (compuesto por 17 artículos y $\alpha=0,92$ ), las interacciones sociales (cinco artículos y $\alpha=0,75$ ) Instrumental (siete artículos y $\alpha=0,82$ ) y hacer frente a los problemas (siete artículos y $\alpha=0,83$ ). El EPSUS-A ha demostrado ser una escala apropiada para la evaluación del apoyo social; se necesitan más estudios pero con el fin de poner de relieve otras formas de validez del instrumento.

Palabras clave: Evaluación psicológica; Pruebas psicológicas; Interacción interpersonal.
\end{abstract}

\section{Introdução}

Atualmente, a avaliação psicológica está presente em diversos contextos de atuação do psicólogo, como, por exemplo, escolas, hospitais, organizações, clínica, esporte, dentre outros (Tavares, 2010). Essa, conforme ressalta Weschler (1999), pode ser definida como um processo de coleta de dados com objetivo de obter maior conhecimento dos sujeitos avaliados, podendo, o psicólogo, para tanto, o psicólogo utilizar-se de diversos recursos, tais como: testes psicológicos, entrevistas e observação dos comportamentos. Especificamente sobre teste psicológico, este pode ser definido como uma medida objetiva e padronizada de amostras do comportamento (Anastasi \& Urbina, 2000). Por sua vez, um instrumento, para ser considerado útil nesse processo deve apresentar evidências que atestem sua validade e confiabilidade, ou seja, é necessário possuir adequadas propriedades psicométricas.

Em relação à definição de validade, Alves, Souza e Baptista (2011) destacam que a mesma está relacionada às evidências acerca do quanto se pode inferir a partir 
dos escores de um instrumento psicológico, podendo ser evidenciada por meio de cinco classificações, ou seja, evidência de validade com base no conteúdo, com base no processo de resposta, com base na estrutura interna, com base na relação com variáveis externas e evidências baseadas nas consequências da testagem. Outra qualidade psicométrica que um teste psicológico deve apresentar é a precisão, também denominada como fidedignidade ou confiabilidade. De acordo com Hogan (2006) esse parâmetro psicométricos está relacionado com o grau de confiança que os escores de um instrumento produzem, podendo ser obtido por intermédio dos procedimentos de teste-reteste, interavaliadores, formas alternadas e consistência interna.

A partir de 2003, por intermédio da criação do Sistema Avaliação de Testes Psicológicos (SATEPSI), muitos estudos foram desenvolvidos no intuito de se produzirem instrumentos psicológicos favoráveis para auxiliar os psicólogos na prática profissional (Anache \& Borges, 2010; Noronha \& Alchieri, 2004). O SATEPSI é um serviço prestado pelo Conselho Federal de Psicologia (CFP) que avalia os instrumentos psicológicos nacionais e, por meio de seu trabalho, é possível constatar se um determinado instrumento psicológico a ser usado no contexto profissional apresenta as condições mínimas quanto aos parâmetros psicométricos, ou seja, se o mesmo pode ser considerado um teste psicológico. A presente pesquisa se insere na temática avaliação psicológica tendo como construto central o suporte social. Em relação a este, embora amplamente estudado no contexto internacional, no Brasil, pesquisas atuais não têm mostrado atenção semelhante, assim como não há registro no SATEPSI de instrumento reconhecido que avalie esse conceito.

O suporte social, enquanto objeto de estudo da Psicologia e de outras ciências relacionadas à saúde, tem sido concebido como importante variável amortecedora ante situações estressantes vivenciadas pelos indivíduos (Casale \& Wild, 2012). Como ressaltam Barth, Schneider e Känel (2010), evidências empíricas apontam que a baixa percepção de suporte social pode ser um dos fatores contribuintes para o desenvolvimento de patologias como depressão, transtornos de ansiedade, indo na contramão da adequada percepção de qualidade de vida e bem-estar psicológico.

Taylor (2011) destaca que o suporte social é considerado um construto multidimensional, uma vez que pode ser percebido em diversas esferas de atuação do indivíduo. A autora também ressalta que a avaliação do suporte social é subjetiva, isto é, depende da percepção, implicando que, por vezes, o apoio ofertado e percebido pelo indivíduo pode ser discrepante. Assim, a avaliação do suporte social historicamente vem sendo realizada com base em propósitos estruturais e funcionais. Por estruturais pode-se entender a avaliação da rede social, ou seja, trata-se de uma análise com características quantitativas, relacionada a número de pessoas percebidas como suportivas e, consequentemente, mensuração de amplitude de rede social. A segunda forma de investigação, funcional, possui características mais qualitativas. Nesses termos, busca avaliar a percepção do acolhimento social por meio de suas possíveis fontes de suporte.

Com base nessas últimas afirmações, cabe destacar a teoria de suporte social proposta por Rodriguez e Cohen (1998), os quais postulam que o mesmo pode ser mensurado por meio de três fontes de suporte, sendo estas: emocional, instrumental e informacional. Por emocional, os autores entendem que diz respeito à percepção, por parte do indivíduo, de afetividade recebida de outras pessoas. Essa fonte de suporte está ligada ao senso de ser amado, apreciado e ter consciência de que as pessoas se preocupam com o indivíduo. A segunda fonte, instrumental, se refere à percepção de auxílios de forma prática, ou seja, relaciona-se a recursos de ordem financeira, tais como emprestar dinheiro ou pagar contas, e também é expressa por meio de comportamentos, como, por exemplo, levar o indivíduo ou alguém de sua família ao médico, preparar refeições em caso de impossibilidade por parte do indivíduo, dentre outros comportamentos. Essa fonte de apoio, de acordo com os autores, proporciona segurança em casos de instabilidades financeiras, assim como pode ser percebida como manifestações de afeto, relacionando-se, em certo ponto, à dimensão emocional. Por sua vez, a terceira fonte de suporte, informacional, diz respeito a receber, de outras pessoas, informações pertinentes em determinadas circunstâncias, geralmente ligadas a ocasiões de vida que remetam à tomada de decisões.

No que tange às avaliações por meio de instrumentos psicológicos, alguns autores convergem com a ideia de que, por conta da característica multifacetada, a definição e operacionalização do construto suporte social se torna complexa. De forma geral, a avaliação do suporte social remete à questão da percepção de recebimento desse apoio e, dessa forma, a mensuração se dá por meio da investigação do quanto as pessoas percebem o apoio vindo de outros indivíduos de sua rede social, podendo em alguns casos, fazer distinção entre essas pessoas, tais como, membros da família, da 
comunidade ou grupos específicos. Outras avaliações, porém, pontuam que o suporte social deve ser avaliado no que tange não só à quantidade de pessoas consideradas suportivas, mas também deve-se mensurar a qualidade de tais interações, ou seja, não somente a percepção do suporte recebido pelos indivíduos, mas também a satisfação com o mesmo (Agneessens, Waege \& Lievens, 2006; Yang, Peek-Asa, Lowe, Heiden \& Foster, 2010).

De acordo com Gonçalves, Pawlowski, Bandeira e Piccinini (2011), no que tange à avaliação do suporte social, no contexto internacional são encontrados diversos instrumentos, quando comparado ao contexto brasileiro. Dentre algumas formas de avaliação do contexto internacional, pode-se destacar o Social Support Questionnaire - SSQ (Sarason, Levine, Basham, \& Sarason, 1983), Perceived Social Support from Friends and Family - PSS-Fr e PSS-Fa (Procidano \& Heller, 1983), a Social Provisions Scale (Cutrona \& Russell, 1987), Multidimensional Scale of Perceived Social Support - MSPSS (Zimet, Dahlem, Zimet \& Farley, 1988), o Medical Outcomes Study - MOS (Sherbourne \& Stewart, 1991), dentre outros.

Já no Brasil, esse número de instrumentos é bastante reduzido, se comparado ao contexto internacional (Gonçalves, Pawlowski, Bandeira \& Piccinini, 2011). Embora não haja nenhum instrumento aprovado pelo Conselho Federal de Psicologia (CFP), ou seja, não há um teste psicológico que mensure o construto psicológico da percepção do suporte social e que possa ser utilizado no contexto de atuação profissional do psicólogo (CFP, 2003; SATEPSI, 2012), existem alguns instrumentos que avaliam o mesmo construto e estão abordados na literatura científica. Entretanto, cabe destacar que a maior parte desses instrumentos é decorrente de adaptações de testes internacionais (Chor, Griep, Lopes \& Faerstein, 2001; Matsukura, Marturano \& Oishi, 2002; Seidl \& Tróccoli, 2006) e que apenas um, até o presente momento, foi construído e validado no Brasil (Faerstein, Lopes, Valente, Plá \& Ferreira, 1999).

De forma mais específica, Matsukura, Marturano e Oishi (2002) adaptaram o Social Support Questionnaire SSQ, de Sarason, Levine, Basham, \& Sarason, (1983). $\mathrm{O}$ instrumento tem objetivo de avaliar a quantidade de pessoas percebidas como suportivas por parte do respondente do questionário, ou seja, é possível constatar a amplitude de rede de apoio social, sendo denominada escala N (SSQ-N), bem como o grau de satisfação com essas pessoas, escala S (SSQ-S). Para tanto, os pesquisadores brasileiros traduziram os 27 itens originais e os aplicaram em uma amostra de 113 mães. Quanto aos resultados, mantiveram no SSQ os 27 itens, assim como na versão internacional, e apresentou confiabilidade teste-reteste adequada.

A Escala de Suporte Social para Pessoas Vivendo com HIV/Aids (Seidl \& Tróccoli, 2006) tem como objetivo mensurar o suporte social em pessoas soropositivas para o HIV. Trata-se de uma escala adaptada de um instrumento canadense, o Social Support Inventory for People who are Positive or Have AIDS (Renwick \& cols., 1999). No estudo de adaptação da escala brasileira, os 54 itens, distribuídos em três dimensões (suporte social emocional, suporte social instrumental e suporte social informacional) foram traduzidos e adequados semanticamente para o português. $\mathrm{O}$ instrumento foi aplicado em uma amostra de 10 pessoas soropositivas, tendo sido possível verificar que a escala brasileira se diferenciou do instrumento canadense, ou seja, a versão brasileira, inicialmente, foi composta por 26 itens, sendo 10 referentes ao suporte instrumental e 16 itens referentes ao suporte emocional (Seidl \& Tróccoli, 2006).

$\mathrm{Na}$ validação por meio da estrutura interna, a escala foi aplicada em uma amostra de 241 pacientes com sorologia positiva para o HIV. Os autores realizaram duas análises fatoriais exploratórias, a primeira com a extração de dois fatores e a segunda adotando o conjunto de itens como unifatorial, sendo que os dois modelos se mostraram satisfatórios, ambos com os mesmos 24 itens. Em relação à análise com dois fatores, os autores denominaram o primeiro como suporte social emocional, composto por 12 itens e confiabilidade alfa de Cronbach $(\alpha)$ de 0,92 , e o segundo fator, suporte instrumental, também composto por 12 itens e $\alpha=0,84$. Em relação à escala unifatorial, o conjunto de 24 itens apresentou confiabilidade $\alpha=0,87$ (Seidl \& Tróccoli, 2006).

Dentre os três instrumentos brasileiros de suporte social adaptados, o que mais apresenta estudos publicados quanto às qualidades psicométricas é o questionário Medical Outcomes Study - MOS (Faerstein, Lopes, Valente, Plá \& Ferreira, 1999). Esse instrumento foi adaptado a partir do questionário de mesmo nome, o Medical Outcomes Study - MOS (Sherbourne \& Stewart, 1991). Para a adaptação, os autores brasileiros traduziram os 19 itens da escala original e realizaram diversos estudos, como, por exemplo, confiabilidade teste-reteste (Griep, Chor, Faerstein \& Lopes, 2003) validade de construto (Griep, Chor, Faerstein, Werneck \& Lopes, 2005; 
Zanini, Verolla-Moura \& Queiroz, 2009). A escala, em sua versão brasileira, ficou composta também por 19 itens, respondidos por meio de uma escala do tipo likert de cinco pontos, e divididos em quatro fatores (interação social, composto por quatro itens e $\alpha=0,94$; apoio emocional/de informação, oito itens e $\alpha=0,95$; apoio material, quatro itens e $\alpha=0,88$ e afetivo, três itens e $\alpha=0,76)$.

No que se refere ao instrumento de suporte social construído e validado no Brasil, Siqueira (2008), em seu estudo, construiu e validou a Escala de Percepção de Suporte Social (EPSS). O instrumento foi construído com base nas três dimensões do suporte social de Rodriguez e Cohen (1998). Na primeira versão da EPSS foram elaborados 12 itens relativos ao suporte emocional, 10 em relação ao suporte instrumental e 10 relacionados ao suporte informacional, totalizando 32 itens. O conjunto de itens foi avaliado por um grupo de 10 doutores em psicologia que analisaram a adequação dos itens com base nas definições de cada dimensão. Do resultado, sete itens não alcançaram o critério de concordância estabelecido e, nesse sentido, três foram eliminados e quatro itens foram redigidos, entretanto, não há informações a respeito de uma nova avaliação dos itens reformulados (Siqueira, 2008).

Ainda de acordo com o mesmo estudo, após a análise teórica dos itens, a escala, composta por 29 itens, respondidos por meio de uma escala do tipo likert de quatro pontos, foi aplicada em 437 pessoas e submetida à validação de construto por meio da análise fatorial. Dos resultados, a EPSS ficou composta por dois fatores, denominados suporte prático (fator 1, composto por 19 itens e confiabilidade de 0,91) e suporte emocional (fator 2, composto por 10 itens e fidedignidade de 0,92).

Essa explanação acerca dos instrumentos de suporte social no contexto internacional, e mais especificamente no âmbito brasileiro, se faz necessária, uma vez que o presente estudo se pautará no referido construto. Como pode ser percebido, no Brasil, a maioria dos instrumentos de suporte social é decorrente de estudos de adaptação e apenas uma escala, até o presente momento, foi construída e validada, porém ainda não há registro no SATEPSI da referida escala. Tendo essa premissa, o presente estudo teve por objetivo a busca por evidência de validade, com base na estrutura interna e precisão por meio do método de consistência interna, para a Escala de Percepção do Suporte Social (versão adulta) - EPSUS-A, um instrumento de avaliação da percepção do suporte social.

\section{Método}

\section{Participantes}

A amostra da pesquisa foi composta por 533 estudantes que cursavam o ensino superior (graduação e pós-graduação), provenientes dos estados de São Paulo $(n=438)$, Minas Gerais $(n=68)$ e Bahia $(n=27)$, com faixa etária variando de 18 a 62 anos $(M=25,6 ; D P=8,2)$, sendo a maioria da amostra do sexo feminino, com 368 $(69 \%)$. No que se refere ao estado civil, do total da amostra, $415(77,9 \%)$ eram solteiros e, em relação à religião, 261 (49\%) eram católicos, seguidos de 44 (8,3\%) evangélicos.

\section{Instrumento}

Escala de Percepção do Suporte Social (versão adulta) - EPSUS-A (Baptista \& Cardoso, 2010).

A EPSUS-A foi construída com base na teoria de suporte social de Rodriguez e Cohen (1998), a qual estipula três possíveis fontes de suporte social. Os itens iniciais foram construídos no ano de 2010 e fizeram parte de uma das etapas do projeto de doutorado do primeiro autor do artigo. A primeira versão da escala ficou composta por 77 itens, respondidos por meio de uma escala likert de quatro pontos (sempre; muitas vezes; poucas vezes; nunca), com pontuação variando entre zero e 231, na qual, quanto maior a pontuação na escala, maior o indício de percepção do suporte social. Por meio de análise semântica dos itens, realizada por dois pesquisadores, esses se agruparam nas três dimensões propostas pelos referidos autores. Nessa mesma direção, 49 agruparam-se na categoria emocional, 16 na dimensão instrumental e 12 itens formaram a fonte de suporte informacional.

\section{Procedimentos}

Após a aprovação deste projeto pelo Comitê de Ética em Pesquisa da Universidade São Francisco (protocolo $\mathrm{n}^{\circ}$ 0240.0.142.000-11), buscou-se a autorização dos professores para a aplicação do instrumento em salas de aula, o qual foi aplicado mediante o consentimento dos universitários, de forma coletiva, com média de aplicação de 15 minutos.

\section{Procedimentos de Análise de dados}

Foi realizada a análise fatorial exploratória da EPSUS-A por meio da extração dos fatores com o modelo de Análise dos Componentes Principais e rotação oblimin. Para a análise da confiabilidade ou 
precisão, foi utilizado o alfa de Cronbach. Nesse sentido, foram analisados os conjuntos de itens que formam os fatores, em separado, assim como análise da escala em sua totalidade.

\section{Resultados}

No primeiro momento, foi realizada uma análise fatorial exploratória no intuito de verificar se o conjunto de 77 itens era passível de fatoração e quantas dimensões essa primeira análise sugeriria. Para tanto, foi utilizado como parâmetros eigenvalues iguais ou maiores que um e cargas fatoriais acima de 0,30. Nessa primeira análise fatorial exploratória, o KMO foi de 0,95 e apresentou como teste de esfericidade de Bartlett, $\chi^{2}=22779,9, \mathrm{p}<0,001$. Nessa análise livre foram retirados 15 fatores, explicando $61,9 \%$ da variância total. Por meio do gráfico de sedimentação dos autovalores foi percebido que havia a possibilidade de configuração em quatro fatores, ao invés de três, como apontado pela teoria adotada. Nesse sentido, uma nova análise foi realizada forçando quatro fatores.

Em um segundo momento, como critérios de exclusão de itens, foram retirados aqueles que se apresentaram em mais de um fator, de conteúdos semelhantes e com cargas fatoriais abaixo de 0,45 . Com relação ao $\mathrm{KMO}$, nessa segunda análise, foi de 0,94 , e o teste de esfericidade de Bartlett apresentou $\chi^{2}=8968,18$ $(\mathrm{df}=666), \mathrm{p}<0,001$. De acordo com Pereira (1999), ao se referir sobre os valores do KMO, destaca que valor acima de 0,80 já é um indicativo de boa adequação dos dados para execução da análise fatorial. A Tabela 1 mostra a variância explicada pelas quatro dimensões encontradas na análise da EPSUS-A, contendo 36 itens, explicando 49,57\% da variância total.

O fator 1 (afetivo) ficou composto por itens relacionados ao suporte de ordem emocional, com o qual $\mathrm{o}$ indivíduo percebe que pode contar de outras pessoas, como, por exemplo, Posso contar com pessoas que (a) "me proporcionam situações agradáveis", (b) "são agradáveis para se conversar", (c) "respeitam minhas opiniões", dentre outros. O fator 2 (interação social) condensou itens relativos aos relacionamentos do sujeito com outros indivíduos, bem como a possibilidade de participação de eventos sociais, como exemplo: Posso contar com pessoas que (a) "me convidam para atividades sociais", (b) "me convidam para atividade de lazer, (c) "me convidam para praticar atividades físicas".

No fator 3 (instrumental), agruparam-se os itens referentes a percepção de suporte de ordem material, tais como: Posso contar com pessoas que (a) "pagam minhas contas quando tenho um problema financeiro", (b) "me fornecem alimentação quando preciso", (c) "ajudam a comprar a medicação quando estou doente". Por último, no fator 4 (enfrentamento de problemas) aglomeraram-se os itens sobre a qualidade da circulação de informações nas interações sociais, assim como a percepção de pessoas concebidas como suportivas e que auxiliam os indivíduos por meio de conselhos ou instruções úteis no processo de tomada de decisões e resolução de conflitos, como pode ser percebido pelos itens: Posso contar com pessoas que (a) "compreendem meus problemas", (b) "escutam meus problemas sem me julgar", (c) "auxiliam em momentos de tomadas de decisões em minha vida", dentre outros.

$\mathrm{Na}$ Tabela 2, são apresentadas as cargas fatoriais do modelo final da EPSUS-A, convertida em 15 iterações. Houve a preponderância de quatro fatores, sendo esses, afetivo (fator 1), interações sociais (fator 2), instrumental (fator 3) e enfrentamento de problemas (fator 4).

Por meio da análise da Tabela 2 percebe-se que os 36 itens da EPSUS-A apresentaram cargas fatoriais acima de 0,45. De acordo com Pasquali (1999), é considerado parâmetro mínimo aceitável para esse tipo de procedimento cargas fatoriais acima de 0,30. A Tabela 3 apresenta a análise de itens por intermédio da correlação item-total, separados por fatores.

Tabela 1. Total de variância explicada

\begin{tabular}{lccc}
\hline Fatores & Eigenvalues & Variância $(\%)$ & Variância Acumulada (\%) \\
\hline Afetivo & 12,94 & 34,99 & 34,99 \\
Interações sociais & 2,04 & 5,53 & 40,52 \\
Instrumental & 1,86 & 5,04 & 45,56 \\
Enfrentamento de problemas & 1,48 & 4,01 & 49,57 \\
\hline
\end{tabular}

Método de Extração: análise dos componentes principais. 
Tabela 2. Cargas fatoriais dos componentes rotados extraídos de análise dos componentes principais e comunalidades da EPSUS-A

\begin{tabular}{|c|c|c|c|c|c|}
\hline & 1 & 2 & 3 & 4 & $\mathrm{~h}^{2}$ \\
\hline EPSUS-A71 & 0,74 & & & & 0,55 \\
\hline EPSUS-A72 & 0,68 & & & & 0,53 \\
\hline EPSUS-A40 & 0,66 & & & & 0,52 \\
\hline EPSUS-A35 & 0,65 & & & & 0,47 \\
\hline EPSUS-A69 & 0,64 & & & & 0,51 \\
\hline EPSUS-A32 & 0,63 & & & & 0,40 \\
\hline EPSUS-A38 & 0,59 & & & & 0,46 \\
\hline EPSUS-A74 & 0,57 & & & & 0,49 \\
\hline EPSUS-A58 & 0,55 & & & & 0,54 \\
\hline EPSUS-A52 & 0,54 & & & & 0,51 \\
\hline EPSUS-A55 & 0,54 & & & & 0,45 \\
\hline EPSUS-A66 & 0,53 & & & & 0,40 \\
\hline EPSUS-A36 & 0,52 & & & & 0,45 \\
\hline EPSUS-A65 & 0,51 & & & & 0,53 \\
\hline EPSUS-A44 & 0,50 & & & & 0,52 \\
\hline EPSUS-A62 & 0,49 & & & & 0,46 \\
\hline EPSUS-A42 & 0,48 & & & & 0,42 \\
\hline EPSUS-A6 & & 0,69 & & & 0,57 \\
\hline EPSUS-A8 & & 0,65 & & & 0,56 \\
\hline EPSUS-A16 & & 0,48 & & & 0,51 \\
\hline EPSUS-A14 & & 0,47 & & & 0,38 \\
\hline EPSUS-A59 & & 0,45 & & & 0,43 \\
\hline EPSUS-A30 & & & 0,76 & & 0,61 \\
\hline EPSUS-A76 & & & 0,74 & & 0,59 \\
\hline EPSUS-A46 & & & 0,67 & & 0,50 \\
\hline EPSUS-A11 & & & 0,63 & & 0,50 \\
\hline EPSUS-A57 & & & 0,58 & & 0,53 \\
\hline EPSUS-A34 & & & 0,49 & & 0,36 \\
\hline EPSUS-A51 & & & 0,46 & & 0,54 \\
\hline EPSUS-A10 & & & & 0,75 & 0,54 \\
\hline EPSUS-A9 & & & & 0,59 & 0,49 \\
\hline EPSUS-A20 & & & & 0,59 & 0,50 \\
\hline EPSUS-A29 & & & & 0,57 & 0,47 \\
\hline EPSUS-A19 & & & & 0,56 & 0,55 \\
\hline EPSUS-A25 & & & & 0,55 & 0,41 \\
\hline EPSUS-A41 & & & & 0,54 & 0,45 \\
\hline Eigenvalues & 12,94 & 2,04 & 1,86 & 1,48 & \\
\hline Variância explicada & 34,99 & 5,53 & 5,04 & 4,01 & \\
\hline $\begin{array}{l}\text { Total de variância } \\
\text { explicada }\end{array}$ & 49,57 & & & & \\
\hline
\end{tabular}

Método de Extração: análise dos componentes principais. Método de rotação: oblimin com normalização Kaiser.
Os valores de correlação item-total considerando cada fator isolado foram expressos na Tabela 3. Cabe destacar que todos os coeficientes foram superiores a 0,30, que, de acordo com a literatura, é o valor mínimo aceitável para esse tipo de análise (Guilford \& Fruchter, 1978). Com relação às análises de correlação com significância estatística entre as dimensões da EPSUS-A e a escala em sua totalidade, obteve-se 0,58 (entre dimensões afetivo e interações sociais), 0,58 (afetivo e instrumental), 0,69 (afetivo e enfrentamento de problemas), 0,93 (afetivo e escala total), 0,41 (interações sociais e instrumental), 0,57 (interações sociais e enfrentamento de problemas), 0,72 (interações sociais e escala total), 0,55 (instrumental e enfrentamento de problemas), 0,76 (instrumental e enfrentamento de problemas) e 0,83 (enfrentamento de problemas e escala total).

A confiabilidade da EPSUS-A, bem como de seus fatores em separado, foi obtida por meio do coeficiente alfa de Cronbach $(\alpha)$. De acordo com Pasquali (1999), os coeficientes de fidedignidade são considerados aceitáveis quando se apresentam acima de 0,70 . Nessa mesma direção, o CFP, por meio do SATEPSI (2004), convenciona como índice mínimo de aceitação 0,60 ; entretanto, considera esse valor como suficiente, e que acima de 0,60 os índices são considerados bons. No caso da EPSUS-A, todos os coeficientes foram acima desses parâmetros. Em acréscimo, o fator 1 (afetivo), composto por 17 itens, apresentou alfa de Cronbach $(\alpha=0,92)$; o fator 2 (interações sociais), composto por cinco itens $(\alpha=0,75)$; o fator 3 (instrumental), composto por sete itens $(\alpha=0,82)$; o fator 4 (enfrentamento de problemas), composto por sete itens $(\alpha=0,83)$. A EPSUS-A, em sua totalidade, ficou composta por 36 itens e com satisfatória confiabilidade $(\alpha=0,94)$.

\section{Discussão}

O processo de construção dos itens da EPSUS-A se baseou no conceito de suporte social de Rodriguez e Cohen (1998). Por intermédio de testes de KMO e esfericidade de Bartlett, foi constatado que o conjunto de 77 itens era passível de fatoração (Pereira, 1999). Por meio da análise dos componentes principais foi percebido que a estrutura de itens apresentou-se distribuída em quatro fatores, assim denominados: afetivo; interações sociais; instrumental e enfrentamento de problemas. Os 36 itens que permaneceram na escala pós-análise fatorial se apresentaram com cargas fatoriais acima de 0,45. Essa informação é contemplada por Pasquali (1999), 
Tabela 3. Correlação item-total por fator

\begin{tabular}{|c|c|c|c|c|c|c|c|}
\hline Fator 1 & $\mathrm{R}$ & Fator 2 & $\mathrm{R}$ & Fator 3 & $\mathrm{r}$ & Fator 4 & $\mathrm{r}$ \\
\hline EPSUS-A71 & 0,68 & EPSUS-A6 & 0,54 & EPSUS-A30 & 0,58 & EPSUS-A10 & 0,58 \\
\hline EPSUS-A72 & 0,66 & EPSUS-A8 & 0,56 & EPSUS-A76 & 0,64 & EPSUS-A9 & 0,57 \\
\hline EPSUS-A40 & 0,62 & EPSUS-A16 & 0,56 & EPSUS-A46 & 0,58 & EPSUS-A20 & 0,61 \\
\hline EPSUS-A35 & 0,61 & EPSUS-A14 & 0,43 & EPSUS-A11 & 0,55 & EPSUS-A29 & 0,59 \\
\hline EPSUS-A69 & 0,64 & EPSUS-A59 & 0,46 & EPSUS-A57 & 0,56 & EPSUS-A19 & 0,65 \\
\hline EPSUS-A32 & 0,56 & & & EPSUS-A34 & 0,46 & EPSUS-A25 & 0,51 \\
\hline EPSUS-A38 & 0,61 & & & EPSUS-A51 & 0,55 & EPSUS-A41 & 0,53 \\
\hline EPSUS-A74 & 0,64 & & & & & & \\
\hline EPSUS-A58 & 0,63 & & & & & & \\
\hline EPSUS-A52 & 0,63 & & & & & & \\
\hline EPSUS-A55 & 0,60 & & & & & & \\
\hline EPSUS-A66 & 0,55 & & & & & & \\
\hline EPSUS-A65 & 0,62 & & & & & & \\
\hline EPSUS-A36 & 0,58 & & & & & & \\
\hline EPSUS-A44 & 0,62 & & & & & & \\
\hline EPSUS-A62 & 0,62 & & & & & & \\
\hline EPSUS-A42 & 0,59 & & & & & & \\
\hline
\end{tabular}

uma vez que, para esse autor, índices fatoriais acima de 0,30 são considerados adequados no processo de análise fatorial.

No que tange às análises dos fatores da EPSUS-A, o primeiro, "afetivo", ficou composto por itens relacionados ao suporte de ordem emocional que o indivíduo percebe e pode contar como vindo de outras pessoas. No que tange à importância da afetividade no estudo do apoio social, Cohen e Pressman (2006) enaltecem que a dimensão emocional desempenha importante papel na saúde mental dos indivíduos.

Para esses autores, a afetividade, se percebida de forma positiva no processo de vinculação dos indivíduos, tende a desempenhar função amortecedora em relação a eventos estressores, bem como sintomatologias de depressão e ansiedade. Nessa mesma direção, o afeto positivo tende a proporcionar aos indivíduos maior senso de pertencimento a uma rede de apoio social, assim como a afetividade pode acarretar maior autoestima. De acordo com Langford, Bowsher, Maloney e Lillis (1997), o suporte emocional está relacionado à percepção do individuo em relação a ser cuidado, apoiado e valorizado por outras pessoas. Essa afirmação também é corroborada por Taylor (2011), a qual destaca que o suporte de ordem emocional possui efeito direto e mais eficaz, quando comparado aos outros tipos de apoio, ou seja, embora todas as fontes de suporte sejam consideradas importantes no processo de pertencimento de grupo, e consequentemente percepção de bem-estar e saúde, a dimensão emocional, por se relacionar a comportamentos que remetam a afetividade, tende a ser concebida como a de maior efeito positivo para os indivíduos.

A dimensão dois, interação social, é composta por itens associados à percepção de possíveis vínculos disponíveis, haja vista que os mesmos se referem a atividades sociais e de lazer. Acerca dessa dimensão, autores como Hupcey (1998) e Langford, Bowsher, Maloney e Lillis (1997) destacam que as relações interpessoais, oferecidas por pessoas ou grupos, são percebidas, na maioria das vezes, de forma benéfica pelos indivíduos, uma vez que estão relacionados a comportamentos e efeitos emocionais positivos. Due e colaboradores (1999) ressaltam, também, que as interações sociais podem proporcionar aos indivíduos senso de integração, minimizando, dessa forma, as tensões sociais ou mesmo relações conflitantes.

O terceiro fator da EPSUS-A, instrumental, diz respeito a percepção de suporte de ordem material e tangível. No que tange a esse fator, Langford, Bowsher, Maloney e Lillis (1997) ressaltam que está relacionado à realização de atividades concretas que 
auxiliam na resolução de problemas, por isso, percebidas como manifestação de suporte. De acordo com Rodriguez e Cohen (1998), a fonte instrumental de suporte social está relacionada a ajudas financeiras, auxílio em relação à saúde, como, por exemplo, levar ao médico, além de ajudas de ordem prática, como oferecimento de moradia e preparar alimentos em caso de impossibilidade do indivíduo. De acordo com os autores, essas atitudes de ordem instrumental são percebidas como manifestações de acolhimento por parte dos sujeitos que as recebem.

Por último, no fator quatro, enfrentamento de problemas, aglomeraram-se os itens sobre a percepção da qualidade da circulação de informações nas interações sociais, bem como auxílio de pessoas, por meio de conselhos ou instruções úteis, no processo de tomada de decisões e resolução de conflitos. A respeito disso, Andrade e Vaitsman (2002) pontuam que o apoio relacionado ao enfrentamento de problemas ocorre quando há percepção de uma postura ativa de incentivo, ou seja, comportamentos de escuta e empatia oferecidos por outras pessoas, que auxiliam na resolução de conflitos. Taylor (2011), por sua vez, destaca que essa fonte de suporte é percebida na medida em que indivíduo recebe informações pertinentes de membros de sua rede social e que são úteis para tomada de decisões em sua vida. Por fim, Rodriguez e Cohen (1998) destacam que esse tipo de apoio é percebido por meio da prestação de informações ou conselhos que auxiliam os indivíduos.

Como pode ser percebido, os resultados da EPSUS-A não corroboraram, em números, as dimensões postuladas por Rodriguez e Cohen (1998); entretanto, cabe destacar que, embora a EPSUS-A tenha apresentado quatro dimensões, duas delas (afetivo e interações sociais) estão intimamente ligadas à definição do fator emocional. Quanto ao fator Instrumental da EPSUS-A, o mesmo corroborou a teoria dos referidos autores. Por fim, a dimensão enfrentamento de problemas diz respeito à obtenção de informações que ajudariam o indivíduo no processo de tomada de decisão, ou seja, corrobora, em grande parte, a dimensão informacional abordada pelos autores.

Com relação às análises de cargas fatoriais, no processo de análise dos componentes principais da EPSUS-A foram solicitadas cargas fatoriais a partir de 0,45 , e os 36 itens que compuseram a escala, após análise, estiveram adequados a esse parâmetro, o que se configura como sendo um nível aceitável de interpretação e que os itens estão bem alinhados (Guilford \& Fruchter, 1978). No que tange às análises de correlação item-total, os dados podem ser considerados satisfatórios, uma vez que apresentaram valores superiores a 0,20 (Likert, 1932). Cabe destacar que, embora a EPSUS-A tenha se apresentado com adequados parâmetros psicométricos, essa não é um instrumento pioneiro na avaliação do suporte social no contexto brasileiro. Por meio de pesquisas em base de dados brasileiras, constatou-se a existência de quatro instrumentos que se propõem a avaliar o referido construto; nesse sentido, torna-se importante a discussão entre a EPSUS-A e os demais instrumentos a fim de focalizar as características de cada, bem como possíveis semelhanças e diferenças.

A primeira escala a ser discutida é a Medical Outcomes Study (MOS), adaptada por Faerstein, Lopes, Valente, Plá e Ferreira (1999), composta por 19 itens, distribuídos em quatro dimensões, "interação social”, "apoio emocional/de informação", "apoio material" e "afetivo". Assim como a EPSUS-A, a MOS também possui quatro dimensões, entretanto o instrumento não foi construído no Brasil, mas sim adaptado. Outra questão a ser destacada é que, em duas dimensões da MOS ("apoio emocional/de informação" e "afetivo"), os itens dizem respeito à fonte de suporte de ordem emocional, assim como não houve separação de fontes diferentes de suporte apontadas pela literatura, sendo essas a emocional e informacional. A EPSUS-A, por sua vez, foi construída no Brasil e apresentou diferenciação entre as dimensões emocional (na EPSUS-A denominada afetivo) e informacional (fator enfrentamento de problemas na EPSUS-A).

O SSQ, adaptado por Matsukura, Marturano e Oishi (2002), apresenta 27 itens e é dividido em duas categorias. A primeira mensura a quantidade de pessoas percebidas como suportivas (SSQ-N) e a segunda avalia o grau de satisfação com essas pessoas (SSQ-S). O instrumento se difere da EPSUS-A em relação ao tipo de mensuração do suporte social, pois enquanto o SSQ tem por objetivo a análise estrutural do suporte social, como por exemplo, amplitude de rede de apoio e satisfação com a mesma, a EPSUS-A tem como propósito a análise funcional do suporte social, ou seja, investigação quanto aos tipos de suportes (emocional, instrumental e informacional) e satisfação com os mesmos, percebido pelo indivíduo em sua rede social.

A Escala de Suporte Social para Pessoas Vivendo com HIV/Aids (Seidl \& Tróccoli, 2006) é composta 24 itens, divididos em dois fatores (suporte social emocional e suporte instrumental). Com base na nomenclatura dos fatores é percebido que a EPSUS-A também avalia essas variáveis e, além dessas, 
contempla itens relacionados a interações sociais e enfrentamento de problemas. Outra diferença está em relação à amostra de aplicação do instrumento, pois enquanto a EPSUS-A pode ser administrada em uma amostra diversificada, a Escala de Suporte Social para Pessoas Vivendo com HIV/Aids possui itens direcionados a essa amostra em específico.

A Escala de Percepção de Suporte Social (EPSS), construída por Siqueira (2008), é composta por 29 itens agrupados em dois fatores, suporte prático e suporte emocional. Assim como a EPSUS-A, a EPSS também foi construída com base nos pressupostos de Rodriguez e Cohen (1998) e também se apresenta como de rápida aplicação, entretanto, as mesmas se diferenciam em aspectos relacionados aos itens avaliados pelas escalas. A EPSS aborda itens relacionados a questões afetivas e de ordem prática (que, segundo a autora, estão relacionados às dimensões instrumental e informacional). Quando comparada com a EPSUS-A, percebe-se que EPSS avalia duas fontes de suporte em um único fator, enquanto a EPSUS-A possui os itens separados em fatores e com adequada confiabilidade. Além disso, a EPSUS-A possui, como diferencial, ser composta de um fator específico de enfrentamento de problemas ou situações que remetam a dúvidas por parte dos indivíduos.

Esse fato pode ser considerado um diferencial, principalmente se for analisado segundo enfoque clínico, pois, como destaca Seidl (2005), o suporte social é uma importante variável a ser trabalhada nos processos clínicos. Ainda de acordo com a autora, o suporte social está relacionado à percepção de estratégias de enfrentamento de problemas e, consequentemente, também tende a estar associado com a melhora do quadro do paciente. Nesse sentido, a EPSUS-A pode ser um instrumento utilizado de forma a verificar avanços no processo psicoterápico à medida que os indivíduos passam a perceber amplitude e satisfação com a rede social disponível, assim como percepção de pessoas que auxiliam no processo de enfrentamento de problemas.

Os índices de confiabilidade da EPSUS-A em sua totalidade, assim como de seus fatores isoladamente, apresentaram-se acima dos parâmetros estabelecidos pela literatura, com valores alfa de Cronbach entre 0,75 e 0,94. De acordo com Pasquali (1999) e Prieto e Muniz (2000), convenciona-se acima de 0,70 como índice de fidedignidade aceitável.

Como salientado, a EPSUS-A apresentou semelhanças e diferenças quando comparada com os instrumentos já existentes na literatura brasileira até então. Também é sabido que este se configurou como um primeiro estudo de evidência de validade para a EPSUS-A, sendo importante dar continuidade a novas investigações, no intuito de evidenciar outras formas de validades para a escala, bem como estudos de normatização.

\section{Considerações finais}

O suporte social tem se mostrado como um importante construto a ser investigado, quando relacionado à percepção de saúde e bem-estar emocional dos indivíduos. Atualmente há certa consistência, obtida por meio de diversos estudos, de que o suporte social pode ser considerado como variável protetora em relação ao processo de manejo do estresse e adoecimento. Dessa forma, sua mensuração pode auxiliar no processo terapêutico, bem como no contexto de atuação social. No Brasil, há algumas iniciativas em relação à construção ou adaptação de instrumentos com tais propósitos, entretanto, estes ainda não estão disponíveis para o uso profissional do psicólogo, ficando restritos apenas para investigações científicas, por meio de pesquisas.

O presente estudo teve como objetivo a busca por evidência de validade para a Escala de Percepção do Suporte Social (EPSUS-A). Por meio da análise fatorial foi possível constatar que o conjunto de itens construído se mostrou satisfatório evidenciando, portanto, validade, com base na estrutura interna para a EPSUS-A. Os dados referentes à confiabilidade da EPSUS-A também foram analisados e se mostraram adequados, de acordo com os parâmetros postulados pela literatura.

A EPSUS-A se mostrou como uma escala adequada para avaliação do suporte social, entretanto novos estudos são necessários no intuito de evidenciar outras formas de validade para o instrumento. Nesse sentido, sugerem-se outros estudos com a aplicação da EPSUS-A juntamente com outros instrumentos, no intuito de verificar se as correlações entre os mesmos se mostrarão coerentes com os dados encontrados na literatura. Estudos com grupos contrastantes se mostram adequados (uma vez que o presente estudo teve como amostra apenas estudantes universitários que trabalhavam) visando verificar se a EPSUS-A diferencia as respostas entre os grupos de forma significativa. Além disso, sugerem-se estudos entre a EPSUS-A e outras escalas que avaliam suporte social, com o intuito de evidência de validade incremental. 


\section{Referências}

Agneessens, F., Waege, H., \& Lievens, J. (2006). Diversity in social support by role relations: a typology. Soc Networks,28, 427-441.

Alves, G. A. S., Souza, M. S., \& Baptista, M. N. (2011). Validade e precisão de testes psicológicos. Em R. A. M. Ambiel, I. S. Rabelo, S. V. Pacanaro, G. A. S. Alves, I. F. A. S. Leme (Orgs.), Avaliação Psicológica: guia de consulta para estudantes e profissionais da psicologia (pp. 109-128). São Paulo: Casa do Psicólogo.

Anache, A. A. \& Borges, F. (2010). Avaliação Psicológica: diretrizes na regulamentação profissional. Em CFP. As políticas do Conselho Federal de Psicologia para a Avaliação Psicológica (pp. 19-30). Brasília: CFP.

Anastasi, A., \& Urbina, S. (2000). Testagem psicológica. (7 $7^{\text {a }}$ ed.) Porto Alegre: Artes Médicas.

Andrade, G. R. \& Vaitsman, J. (2002). Apoio social e redes: conectando solidariedade e saúde. Ciên Saúde Colet, 7, 925-934.

Baptista, M. N. \& Cardoso, H. F. (2010). Escala de Percepção do Suporte Social (versão adulta) - EPSUS-A. Relatório técnico. Universidade São Francisco, Itatiba.

Barth, J., Schneider, S. \& Känel, R. (2010). Lack of social support in the etiology and the prognosis of coronary heart disease: A systematic review and meta-analysis. Psychosomatic Medicine 72, 229-238.

Casale, M. \& Wild, L. (2012). Effects and processes linking social support to caregiver health among HIV/ AIDS-affected carer-child dyads: a critical review of the empirical evidence. Aids and Behavior. Disponível em: http://rd.springer.com/article/10.1007/ s10461-012-0275-1. Acesso em: dez. 2012.

Chor, D., Griep, R. H., Lopes, C. S. \& Faerstein, E. (2001). Medidas de rede e apoio social no Estudo Pró-Saúde: pré-testes e estudo piloto. Cadernos de Saúde Pública, 17, 887-896.

Cohen, S. \& Pressman, S. D. (2006). Positive Affect and Health. Association for Psychological Science, 15(3), 122-125.

Conselho Federal de Psicologia-CFP. (2003). Resolução $n^{0}$ 002/2003 [On-line]. Disponível em: http:// www.pol.org.br. Acesso: 10 nov. 2011.
Cutrona, C. E. \& Russell, D. (1987). The provision of social relationships and adaptation to stress. Em Jones, W. H. \& Perlman, D. (Orgs.). Advances in personal relationships. (pp 37-67). Greenwich, CT.

Due, P., Holstein, B., Lund, R., Modvig, J. \& Avlund, K. (1999). Social relations: network, support and relational strain. Soc. Sci. Med., 48, 661-673.

Faerstein, E., Lopes, C. S., Valente, K., Plá, M. A. S. \& Ferreira, M. B. (1999). Pré-testes de um questionário multidimensional autopreenchível: a experiência do Estudo Pró-Saúde. Physis - Revista de Saúde Coletiva, 9, 117-130.

Gonçalves, T. R., Pawlowski, J., Bandeira, D. R. \& Piccinini, C. A. (2011). Avaliação de apoio social em estudos brasileiros: aspectos conceituais e instrumentos. Ciência \& Saúde Coletiva, 16(3), 1755-1769.

Griep, R. H., Chor, D., Faerstein, E., Werneck, G. L., \& Lopes, C. S. (2005).Validade de constructo de Escala de Apoio Social do Medical Outcomes Study adaptada para o português no Estudo Pró-Saúde. Caderno de Saúde Pública, 21(3), 703-714.

Griep, R. H., Chor, D., Faerstein, E., \& Lopes, C. (2003). Apoio social: confiabilidade teste-reteste de escala no Estudo Pró-Saúde. Caderno de Saúde Pública, 19(2), 625-634.

Guilford, J. P. \& Fruchter, B. (1978). Fundamental statistic in psychology and Education. (6 $\left.6^{\mathrm{a}} \mathrm{ed}.\right)$. New York: McGraw-Hill.

Hogan, T. P. (2006). Introdução à prática de testes psicológicos. Rio de Janeiro: LTC.

Hupcey, J. E. (1998). Clarifying the social support theoryresearch linkage. J. Advan. Nurs., 27, 1231-1241.

Langford, C. P., Bowsher J., Maloney, J. P., \& Lillis, P. P. Social support: a conceptual analysis. J. Advan. Nurs., 25, 95-100.

Likert, R. (1932). A technique for the measurement of attitudes. Archives of Psychology, 140(2), 5-53.

Matsukura, T. S., Marturano, E. M. \& Oishi, J. (2002). O Questionário de Suporte Social (SSQ): estudos da adaptação para o português. Revista Latino-americana de Enfermagem, 10(5), 675-681.

Noronha, A. P. P. \& Alchieri, J. C. (2004). Conhecimento em avaliação psicológica. Revista Estudos de Psicologia, 21(1), 43-52. 
Pasquali, L. (1999). Instrumentos psicológicos: manual prático de elaboraşão. Brasilia: LabPAM \& IBAPP.

Pereira, J. C. R. (1999). Análise de dados qualitativos: estratégias metodológicas para as ciências da saúde, bumanas e sociais. São Paulo: EDUSP;FAPESP.

Prieto, G. \& Muniz, J. (2000). Un modelo para evaluar la calidad de tests usados na Espanha. Disponível em: http:/ / www.cop.es/vernumero.asp?id=41. Acesso em: dez. 2009.

Procidano, M. E. \& Heller, K. (1983). Measures of perceived social support from friends and from family: three validation studies. American Journal of Community Psychology, 11, 1-24.

Renwick, R., Halpen, T., Rudman, D. \& Friedland, J. (1999). Description and validation of a measure of received support specific to HIV. Psychological Reports, 84, 663-673.

Rodriguez, M. S. \& Cohen, S. (1998). Social support. Encyclopedia of menthal health. 3. New York: Academic Press.

Sarason, I. G., Levine, H. M., Basham, M. B. \& Sarason, B. R. (1983). Assessing social support: the social support questionnaire. Journal of Personality and Social Psychology, 44(1), 127-139.

SATEPSI (2004). Avaliação dos testes psicológicos. [On-line]. Disponível em: http://www.pol.org.br. Acesso em: dez. 2012.

SATEPSI (2012). Sistema de Avaliação de Testes Psicológicos. [On-line]. Disponível em: http://www2.pol.org. br/satepsi/sistema/admin.cfm. Acesso em: dez. 2012.

Seidl, E. M. F. (2005). Enfrentamento, aspectos clínicos e sociodemográficos de pessoas vivendo com HIV/AIDS. Psicologia em Estudo 10(3), 421-429.

Seidl, E. M. F. \& Tróccoli, B. T. (2006). Desenvolvimento de Escala para Avaliação do Suporte Social em HIV/AIDS. Psicologia: Teoria e Pesquisa, 22(3), 317-326.

Sherboune, C. D. \& Stewart, A. L. (1991). The MOS Social Support Survey. Social Science and Medicine, 38, 705-714.

Siqueira, M. M. M. (2008). Construção e validação da escala de percepção de suporte social. Psicologia em Estudo, 12(2), 381-388.

Tavares (2010). Da ordem social da regulamentação da Avaliação Psicológica e do uso dos testes. Em Conselho Federal de Psicologia. Avaliação Psicológica: diretrizes na regulamentação da profissão. (pp. 31-56). Brasília: Conselho Federal de Psicologia.

Taylor, S. E. (2011). Social support. Em Friedman S. H. The Oxford Handbook of Health Psychology. Ed. Howard S. Friedman.

Wechsler, S. M. (1999). Guia de procedimentos éticos para a avaliação psicológica. Em M. Wechsler \& R. S. L. Guzzo (Orgs.). Avaliação Psicológica: perspectiva internacional (pp. 133-141). São Paulo: Casa do Psicólogo.

Yang, J., Peek-Asa, C., Lowe, J. B., Heiden, E. \& Foster, D. T. (2010). Social support patterns of collegiate athletes before and after injury. Journal of Athletic Training, 45(4), 372-379.

Zanini, D. S., Verolla-Moura, A., \& Queiroz, I. P. A. R. (2009). Apoio social: aspectos da validade de construto em estudantes universitários. Psicologia em Estudo, 14(1), 195-202,

Zimet, G. D., Dahlem, N., Zimet, S. \& Farley, G. K. (1988). The Multidimensional Scale of Perceived Social Support. The Journal of Personality Assessment, 52(1), 30-41.

Recebido em: 08/08/13

Reformulado em: 31/03/2014

Aprovado em: 15/04/2014 
Sobre os autores:

Hugo Ferrari Cardoso é doutor em Psicologia pela Universidade São Francisco (USF) e docente do curso de Psicologia da Universidade Estadual Paulista "Júlio de Mesquita Filho" (Unesp/Bauru/SP).

E-mail: hfcardoso@fc.unesp.br

Makilim Nunes Baptista é doutor pelo Departamento de Psicologia Médica e Psiquiatria da Escola Paulista de Medicina, Universidade Federal de São Paulo, bolsista de produtividade pelo CNPq docente do Programa de PósGraduação Stricto Sensu em Psicologia da Universidade São Francisco - USF/Itatiba/SP.

E-mail: makilim.baptista@saofrancisco.edu.br

Contato com os autores:

Rua Alexandre Rodrigues Barbosa, 45

Centro, Itatiba - São Paulo

CEP: 13251-900 